\title{
Correlations between Somatotype and Temperament in Pre-School Twins
}

\author{
Man Zhao, Y.-L. Li ${ }^{*}$, Y.-N, Zheng \\ Institute of Life Science and Technology, Inner Mongolia Normal University, Hohhot, Inner Mongolia, China
}

(*liyuling137@163.com)

\begin{abstract}
To analyze the associations between somatotype and temperament in pre-school children, 124 pairs of 3- to 7-year-old twins were enrolled in. The somatotype components were calculated by Heath-Cater method, while Behavioral Style Questionnaire (BSQ) was used to evaluate child temperament. The results were as follows: There's no correlation between somatotype categories distribution and temperament styles of pre-school twins $(P>0.05)$. The correlation coefficients between ectomorphy and adaptability and emotion were significant $(P<0.01)$. Endomorphic component had negative correlation with activity level, approach/withdrawal, adaptability and persistence $(P<0.01)$. In conclusion, somatotype and temperament showed somewhat associations in pre-school twins.
\end{abstract}

Key words - somatotype, temperament, correlations, pre-school children, twins

\section{学龄前双生子儿童体型与气质的相关性研究 赵曼, 李玉玲*, 郑玉娜 \\ 内蒙古师范大学生命科学与技术学院, 呼和浩特, 内蒙古, 中国}

摘 要 为了分析学龄前双生子儿童体型与气质的相关性, 采用 Heath-Cater 体型法及 Cary 系列问卷对 124 对 3-7 岁双生子儿童 体型及气质进行测评。结果发现: 学龄前双生子儿童体型类型与气质类型的分布无相关性 $(P>0.05)$; 体型类型中的外胚层型与适应性、 情绪本质具有极显著的相关关系 $(P<0.01)$; 体型三因子中的内因子与活动水平、趋避性、适应性及坚持性呈明显的负相关关系 $(P<0.05)$ 。 总的来说, 学龄前双生子儿童体型与气质具有一定的相关性。

关键词 体型, 气质, 相关性, 学龄前儿童, 双生子

体型是人体骨骼、肌肉、体脂、身材等的综合反应, 对青少年的生理机能、运动素质、心理情感、气质发展及 职业选择意向都有一定影响[1]。早在 1918 年, 德国精神病 学家和心理学家 E.Kretschmer 就曾在他的《体型与性格》 一书中提出了气质的体型说[2]。美国心理学家 Sheldon 进 一步研究发现体型与气质的相关系数高达 $80 \%$ 左右[3]。目 前, 我国尚未见双生子儿童体型与气质的相关性研究。因 此, 本文对学龄前双生子儿童的体型与气质进行了测评, 初步探讨儿童体型与气质的相关性, 以便为双生子家长提 供一定的教养指导。

基金项目：国家自然科学基金项目(31260265); 国家自然科学基金项目 (30960167) 基金项目: 国家自然科学基金项目 (31260265); 国家自然科 学基金项目 (30960167); 内蒙古师范大学研究生科研创新基金项目 (CXJJS12046)
1. 对象与方法

1.1 对象

以幼儿园为单位, 在呼和浩特市和包头市募集到双生 子 248 人, 平均年龄为 $5.55 \pm 1.29$ 岁。其中, 同卵双生子 （monozygotic twins, MZ) 94 人, 异卵双生子 (dizygotic twins, DZ) 154 人。该研究获北京大学医学部伦理委员会 批准。

\section{2 方法}

\subsection{1 卵型鉴定}

异性别双生子为异卵双生子。同性别双生子通过提取 
口腔上皮细胞提取儿童 DNA, 采用 ABI 公司的 AMPFS(TM) STR Sinofiler 试剂盒, 复合扩增 16 个 STR (D8S1179、 D21S11、D7S820、CSF1PO、D3S1358、D12S391、D13S317、 D16S539、D2S1338、D19S433、vWA、D6S1043、D5S818、 D18S51、FGA 和性别标记 Amelogenin）位点一致性进行 卵型鉴定，可靠性达 $99.99 \%$ 以上。

\subsection{2 测量工具}

按 Heath-Cater 体型法测量双生子身高、体重、上臂紧 张围、小腿围、肱骨远端宽、股骨远端宽、肱三头肌皮褶、 肩胛下皮褶、髂前上棘皮褶、小腿腓位皮褶等 10 项指标, 计算各体型因子值 $[1,4]$ 。

采用中国标化 Carey 系列气质评价量表: 3-7 岁儿童气 质问卷 (Behavioral Style Questionnaire, BSQ) 对儿童气质 进行评价, 该问卷由家长填写, 使用儿童气质评价软件得 出气质 9 个维度得分及气质类型。

\section{3 统计分析}

采用 SPSS11.0 软件进行一般性统计分析。

\section{2. 结果}

\section{1 双生子儿童体型类型及气质类型分布}

将儿童气质类型划分为 3 种类型, 即易养型、难养型、 启动缓慢型; 体型划分为 4 种类型, 即内胚层型、中胚层 型、外胚层型及混合型。表 1 示双生子儿童体型类型及气 质类型的人数分布。卡方检验结果显示, 双生子儿童气质 类型与体型类型的分布无相关性 $\left(\chi^{2}=6.256, \mathrm{P}>0.05\right)$ 。

表 1 不同体型在不同气质类型中的人数分布

\begin{tabular}{lcccc}
\hline & 内胚层型 & 中胚层型 & 外胚层型 & 混合型 \\
\hline 易养型 & 3 & 135 & 12 & 48 \\
难养型 & 0 & 20 & 5 & 12 \\
启动缓慢型 & 0 & 7 & 2 & 4 \\
合计 & 3 & 162 & 19 & 64 \\
$\chi^{2}$ & \multicolumn{5}{r}{$\begin{array}{l}0.256 \\
P\end{array}$} \\
\hline
\end{tabular}

\section{2 儿童体型类型与气质维度的相关分析}

表 2 示双生子儿童体型类型与气质各维度的相关性分 析, 结果表明外胚层体型与气质维度中的适应性及情绪本 质均存在极显著的相关关系 $(\mathrm{P}<0.01)$ 。SNK 两两比较可知, 内胚层型儿童的趋避性得分显著低于其他各组 $(\mathrm{P}<0.05)$ 。

表 2 双生子儿童体型类型与气质维度的相关性分析 $(\bar{X} \pm S)$

\begin{tabular}{lcccccc}
\hline & $\begin{array}{c}\text { 内胚层型 } \\
(\mathrm{n}=3)\end{array}$ & $\begin{array}{c}\text { 中胚层型 } \\
(\mathrm{n}=162)\end{array}$ & $\begin{array}{c}\text { 外胚层型 } \\
(\mathrm{n}=19)\end{array}$ & $\begin{array}{c}\text { 混合型 } \\
(\mathrm{n}=64)\end{array}$ & $\mathrm{F}$ & $P$ \\
\hline 活动水平 & $3.43 \pm 0.09$ & $3.49 \pm 0.54$ & $3.57 \pm 0.67$ & $3.35 \pm 0.56$ & 1.218 & 0.304 \\
节律性 & $2.67 \pm 0.11$ & $2.81 \pm 0.64$ & $2.83 \pm 0.52$ & $2.74 \pm 0.48$ & 0.242 & 0.867 \\
趋避性 & $2.33 \pm 0.38$ & $3.01 \pm 0.62$ & $3.20 \pm 0.53$ & $2.95 \pm 0.62$ & 1.984 & 0.117 \\
适应性 & $2.72 \pm 0.19$ & $2.75 \pm 0.55$ & $3.08 \pm 0.49$ & $2.58 \pm 0.62$ & 3.960 & $0.009 * *$ \\
反应强度 & $4.58 \pm 0.47$ & $4.26 \pm 0.67$ & $4.41 \pm 0.88$ & $4.13 \pm 0.66$ & 1.201 & 0.310 \\
情绪本质 & $2.92 \pm 0.22$ & $2.87 \pm 0.56$ & $3.24 \pm 0.59$ & $2.60 \pm 0.71$ & 6.336 & $0.000 * *$ \\
坚持性 & $2.70 \pm 0.10$ & $2.84 \pm 0.62$ & $2.87 \pm 0.53$ & $2.67 \pm 0.57$ & 1.488 & 0.218 \\
注意分散度 & $4.43 \pm 0.15$ & $3.80 \pm 0.72$ & $3.88 \pm 0.80$ & $3.63 \pm 0.64$ & 1.988 & 0.116 \\
反应阈 & $3.97 \pm 0.23$ & $3.91 \pm 0.50$ & $4.02 \pm 0.53$ & $3.79 \pm 0.47$ & 1.452 & 0.228 \\
\hline 注. ** $P<0.01$ & & & & & &
\end{tabular}

\section{3 儿童体型因子与气质维度的相关性分析}

运用 Person 相关对儿童体型因子与气质各维度之间进 行相关性分析, 研究结果（表 3) 显示, 体型三因子中的 内因子与活动水平、趋避性、适应性及坚持性均呈显著的 负相关关系 $(\mathrm{P}<0.05)$, 而其他体型因子与气质各维度的相 关系数无统计学意义。

表 3 双生子儿童气质维度与体型因子的相关性分析

\begin{tabular}{llll}
\hline & 内因子 & 中因子 & 外因子 \\
\hline 活动水平 & $-0.130 *$ & 0.032 & 0.020 \\
节律性 & 0.034 & 0.046 & -0.074 \\
趋避性 & $-0.135 *$ & 0.036 & -0.036 \\
适应性 & $-0.166 * *$ & -0.070 & 0.066 \\
反应强度 & 0.023 & -0.064 & 0.003 \\
情绪本质 & -0.085 & -0.014 & 0.009 \\
坚持性 & $-0.168 * *$ & 0.072 & 0.018 \\
注意分散度 & -0.040 & 0.048 & 0.016 \\
反应阈 & -0.067 & -0.030 & 0.020 \\
\hline 注: $* P<0.05, \quad * * P<0.01$ & &
\end{tabular}




\section{3. 讨论}

体型与气质相关学说一直存在争议, 但多数研究结果 表明两者存在一定的相关性[3,5,6]。本文首次对学龄前双生 子儿童体型与气质间的相关关系进行了报道, 研究结果亦 发现体型与气质具有一定的相关性。本研究中, 儿童体型 类型与气质类型分布不具相关性, 但体型类型与气质维度 存在一定的相关关系, 如外胚层型与适应能力及情绪本质。 进一步分析体型因子与气质维度的相关性可知, 内因子与 儿童的活动水平、趋避性、适应性、坚持性等呈显著负相 关, 即儿童的脂肪含量对气质的影响较大。该结果与 Carey[5]等对气质与体型的相关性研究结果一致, 他们认为 儿童气质与肥胖有一定的相关性, 脂肪含量 (内因子) 高 的人, 活动起来相对困难, 故活动能力较差, 但善于交际, 因此适应能力相对较强, 而且能够坚持完成某项任务。此

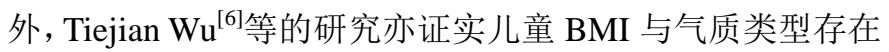
相关关系; 但刘红辉 ${ }^{[7]}$ 在对学龄前儿童气质的相关因素分 析中则指出儿童体型对气质没有影响。

本研究的不足之处首先在于单纯初步分析了双生子儿 童体型与气质间的相关关系, 双生子作为一类特殊人群, 其气质与体型间的相关性与单胎儿童结果相比是否具有一 致性等问题仍未可知, 因此今后有必要进行单、双胎儿童 气质与体型间的比较研究。其次, 样本量有限, 应扩大双 生子样本进一步证实研究结果。

\section{参考文献(References)}

[1] Ji ChengYe. Comparative study on the adolescents' somatotype between China and Japan. Chinese Journal of Preventive Medicine, 1991, 25 (2): 95-98.

[2] Guo Zhengming, Xiao Yuequn, Zhang Bing. Study on the correlation between temperament types and three different somatotypes of college students. Human Guiding Journal of TCMP, 2002, 8 (10):632.

[3] Sheldon WH, Lewis NDC, Tenney AM. Psychotic patterns and physical constitution. In Schizophrenia, Current Concepts and Research, ed. D.V.Siva Sanker. Hicksville, New York: PJD Publications. 1969, pp. 839-911.

[4] Carter JEL and Heath BH. Somatotyping-Development and Applications. Cambridge: Cambridge University Press, 1990: 367-376.

[5] Carey WB, Hegvik RL, and Mc Devitt SC. Temperamental factors associated with rapid weight gain and obesity in middle childhood. Journal of Developmental and Behavioral Pediatrics, 1988, 9 (4):194-198.

[6] Tiejian Wu, Wallance E, Dixon Jr, et al. Joint Effects of Child Temperament and Maternal Sensitivity on the Development of Childhood Obesity. Matern Child Heath J, 2011, 15: 469-477.

[7] Liu Honghui. Analysis of temperament style and the interrelated factors of pre-school age infant in YueLu section of Changsha. Changsha: Central North University, 2007. 